University of Wollongong

Research Online

Faculty of Engineering - Papers (Archive)

Faculty of Engineering and Information

Sciences

15-5-2005

\title{
Vector characterization of soft magnetic materials
}

Z. W. Lin

University of Technology, Sydney

H. W. Lu

University of Technology, Sydney

J. G. Zhu

University of Technology, Sydney

J. J. Zhong

University of Technology, Sydney

Xiaolin Wang

University of Wollongong, xiaolin@uow.edu.au

See next page for additional authors

Follow this and additional works at: https://ro.uow.edu.au/engpapers

Part of the Engineering Commons

https://ro.uow.edu.au/engpapers/164

\section{Recommended Citation}

Lin, Z. W.; Lu, H. W.; Zhu, J. G.; Zhong, J. J.; Wang, Xiaolin; and Ding, Shichao: Vector characterization of soft magnetic materials 2005.

https://ro.uow.edu.au/engpapers/164

Research Online is the open access institutional repository for the University of Wollongong. For further information contact the UOW Library: research-pubs@uow.edu.au 
Authors

Z. W. Lin, H. W. Lu, J. G. Zhu, J. J. Zhong, Xiaolin Wang, and Shichao Ding 


\title{
Vector characterization of soft magnetic materials
}

\author{
Z. W. Lin, ${ }^{\text {a) }}$ H. W. Lu, J. G. Zhu, and J. J. Zhong \\ Faculty of Engineering, University of Technology, Sydney, P.O. Box 123 Broadway, \\ New South Wales 2007, Australia
}

\author{
X. L. Wang \\ Institute for Superconducting and Electronic Materials, University of Wollongong, Wollongong, \\ New South Wales 2522, Australia
}

S. Y. Ding

Department of Physics, Nanjing University, Nanjing, People's Republic of China, 210093

(Presented on 8 November 2004; published online 17 May 2005)

\begin{abstract}
A three-dimensional (3D) magnetic property testing system has been completed and successfully used to measure 3D hysteresis loci of soft magnetic material. This paper presents the techniques to characterize soft magnetic materials under 3D magnetic excitations in detail. Using three couples of excitation coils controlled by a computer to generate the magnetic fields in three orthogonal axes, various types of flux density loci, such as circular or elliptical rotating vectors of flux density with any given orientations in 3D space, can be obtained. Based on 3D finite element analysis and a comparative study, a sandwich arrangement comprising a sample, guard pieces, and search coils with double-layer structure was proposed. Compared with the conventional surface search coils, this arrangement can significantly improve the accuracy of measurement. The comprehensive calibration process and the experimental results of the 3D hysteresis loci of soft magnetic composite materials are also presented. (C) 2005 American Institute of Physics. [DOI: 10.1063/1.1855571]
\end{abstract}

\section{INTRODUCTION}

Hysteresis curves, as a comprehensive means for characterizing magnetic materials, are determined by the complex interplay of magnetic domain wall motion and rotation of local magnetization vectors. Studying these curves will provide clues to elaborate complicated physical phenomena in magnetic materials. One and two-dimensional measurements are widely employed for characterization of magnetic materials. ${ }^{1-9}$ However, they provide insufficient information in describing the actual magnetization mechanisms. The presence of various forms of anisotropy, of magnetocrystalline, and magnetoelastic indicates that physically based models should rely on a treatment of the magnetization process in more detail. ${ }^{10}$ In all cases, the nature of the magnetization process suggests that vector hysteresis models should be the starting point on quite general grounds. Similar conclusions have been drawn in some engineering applications, where the applied field has a vectorial variation over time. For example, the detailed description of the vectorial constitutive relationship in electrical machines is necessary in order to obtain reliable predictions of the rotating flux patterns and the rotational core losses.

In order to gain a complete understanding and modeling of vector magnetization, which is very important for optimum design of novel electromagnetic devices, it is essential to measure three-dimensional (3D) magnetic properties of soft magnetic materials. This paper presents in detail the construction and calibration of a $3 \mathrm{D}$ magnetic property testing

\footnotetext{
a) Author to whom correspondence should be addressed; Electronic mail:
} jacklin@eng.uts.edu.au system. The experimental results of 3D hysteresis loci of the soft magnetic composite material will also be presented and discussed.

\section{3D TESTING SYSTEM}

The system consists of a 3D yoke to guide the magnetic fluxes along the orthogonal $X, Y$, and $Z$ directions, three groups of excitation coils which are wound around the legs of the 3D yoke and controlled by a feedback control system, a sample house located at the center of the testing system, and a computer system for data acquisition and processing. The control system comprises a control circuit unit, a control program, and three high power amplifiers for the $X, Y$, and $Z$ directions, respectively. In this way, various loci, such as circular or elliptical, of rotating flux density vectors in the sample can be obtained.

Six magnetic field $H$ search coils are attached to a cubic sample to measure the sample surface field since tangential component of the magnetic field strength is continuous without applied current flowing at the sample surface. The cubic sample, six magnetic field $H$ search coils, and six guard pieces were integrated into the sample house. Based on 3D finite element analysis and a comparative study, a sandwich arrangement comprising the sample, search coils, and guard pieces is proposed, shown in Fig. 1. The two opposite $H$ coils for the same component are connected in series. Six $H$ coils are glued together to form an $H$ sensing box. The three components of the magnetic flux density in the sample are determined by three orthogonal $B$ coils wound around the sample.

Figure 2 shows the finite element simulation result of the magnetic field distribution around half sample with and with- 

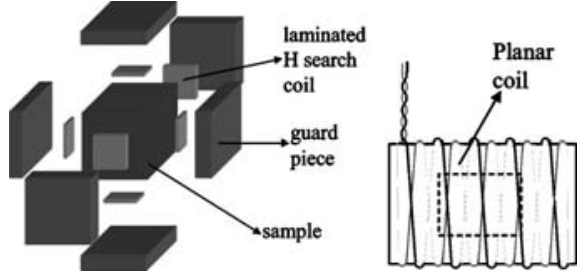

FIG. 1. Arrangement of sample, $H$ coils, and guard pieces (left) and structure of the $H$ coil (right).

out the guard pieces when a dc magnetic field is applied along the $X$ axis. It is clearly shown that with the presence of the magnetic guard pieces, the $X$ component of the magnetic field at the positions where the $H$ coils are located for the $X$ component are more uniform and closer to the field inside the sample, in comparison with the case without the guard pieces. Therefore, adoption of the guard pieces can significantly improve the accuracy of measurement of the sample surface field. Another advantage is the reduction of the air gap between the magnetization pole and the sample, which significantly increases the reluctance of the magnetic circuit, and hence the excitation current required to magnetize the sample can be reduced. It should be noted that the guard pieces must be carefully cut from the sample material and arranged in the same magnetic orientation as the sample.

\section{CALCULATION OF MAGNETIC FIELD PARALLEL TO SAMPLE SURFACE}

The $H$ coils must be calibrated to eliminate the system errors before they can be employed to measure the magnetic field components. The calibration is performed in a long solenoid, which can generate a uniform unidirectional magnetic field. The surface of the coil is set to be parallel to the generated field. The coil constant can be determined as shown in the following.

It must be noted that each of three components of the time varying magnetic field will induce an electromotive force (emf) in an individual $H$ coil, and the resultant emf is a superposition of three emfs. Applying a uniform ac magnetic field $H$ in parallel to the $X$ axis of the $H$ sensing box, the induced emfs from $H$ box for the $X, Y$, and $Z$ axes, $U_{x}^{x}, U_{y}^{x}$, and $U_{z}^{x}$, can be measured and governed by the Faraday induction law as

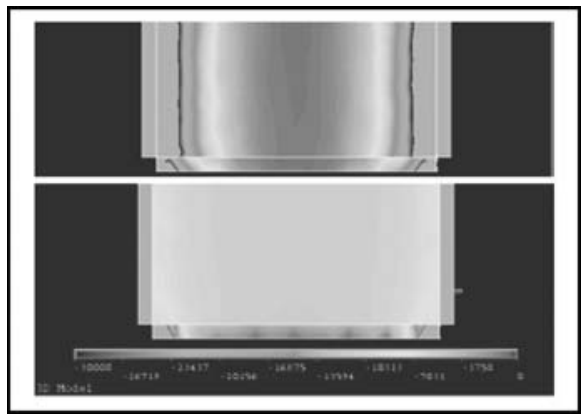

FIG. 2. Magnetic field distributions around half sample without (top) and with guard pieces (bottom).

$$
U_{i}^{x}=-\mu_{0} K_{i}^{x} \frac{d H}{d t} \quad(i=x, y, z)
$$

One can readily derive the coil constant $K_{i}^{x}$. Similarly, one can obtain $K_{i}^{y}$ and $K_{i}^{z}$.

In experiment, when the magnetic field to be measured, $\mathbf{H}=\left(H_{x}, H_{y}, H_{z}\right)$, is not parallel to any axis of $H$ box, the measured emfs in the $H$ coils, $E_{x}, E_{y}$, and $E_{z}$, can be expressed as

$$
\begin{aligned}
& E_{x}=-\mu_{0}\left(K_{x}^{x} \frac{d H_{x}}{d t}+K_{x}^{y} \frac{d H_{y}}{d t}+K_{x}^{z} \frac{d H_{z}}{d t}\right), \\
& E_{y}=-\mu_{0}\left(K_{y}^{x} \frac{d H_{x}}{d t}+K_{y}^{y} \frac{d H_{y}}{d t}+K_{y}^{z} \frac{d H_{z}}{d t}\right), \\
& E_{z}=-\mu_{0}\left(K_{z}^{x} \frac{d H_{x}}{d t}+K_{z}^{y} \frac{d H_{y}}{d t}+K_{z}^{z} \frac{d H_{z}}{d t}\right) .
\end{aligned}
$$

By solving Eqs. (2)-(4), the magnetic field to be measured, $\mathbf{H}=\left(H_{x}, H_{y}, H_{z}\right)$, can be obtained.

When the sample is placed into the $H$ sensing box and subjected to an external magnetic field, the $H$ coils attached to the sample surface are used to measure the component parallel to the surface. However, if the coil is wound in single or odd number of layers, the perpendicular component can also contribute to the reading of the emf. This introduces a significant error in the calculation of $\mathbf{H}$ using Eqs. (2)-(4). To eliminate this perpendicular component contribution, the $H$ coil must be wound uniformly in double or even number of layers with exactly equal number of turns, as illustrated in Fig. 1. Such double-layer structure is also applied to the $B$ coils wound around the sample. However, the calibration process reveals that even the double-layer $H$ coils still couple with the strong perpendicular component. Beware that this perpendicular component cannot be measured by the other $H$ coils since this perpendicular component is not equal to the parallel component at the rounding/side surfaces. This perpendicular component must be measured and taken into account when using Eqs. (2)-(4). The perpendicular component to the surface can be measured using a planar coil attached to the sample surface. In this study, the planar coils are imbedded into the $H$ coils, as shown in Fig. 1. Incorporating the readings of the planar coils into Eqs. (2)-(4), one obtains

$$
\begin{aligned}
& E_{x}=-\mu_{0}\left(K_{x}^{x} \frac{d H_{x}}{d t}+K_{x}^{y} \frac{d H_{y}}{d t}-\frac{K_{x}^{z}}{\mu_{0} N_{x}} V_{x}\right), \\
& E_{y}=-\mu_{0}\left(-\frac{K_{y}^{x}}{\mu_{0} N_{y}} V_{y}+K_{y}^{y} \frac{d H_{y}}{d t}+K_{y}^{z} \frac{d H_{z}}{d t}\right), \\
& E_{z}=-\mu_{0}\left(K_{z}^{x} \frac{d H_{x}}{d t}-\frac{K_{z}^{y}}{\mu_{0} N_{z}} V_{z}+K_{z}^{z} \frac{d H_{z}}{d t}\right),
\end{aligned}
$$

where $V_{x}, V_{y}$, and $V_{z}$ are the emfs across the planar coils embedded in the $H_{x}, H_{y}$, and $H_{z}$ coils, and $N_{x}, N_{y}$, and $N_{z}$ are the planar coil constants determined by the calibration process which is same as that for the $H$ sensing box. Therefore, 

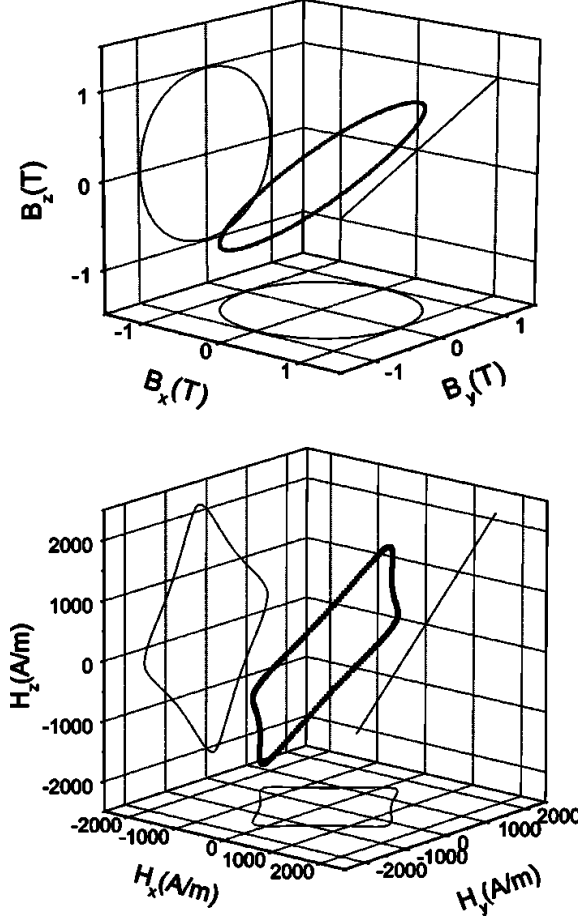

FIG. 3. The loci of vector of magnetic flux density (top) and field strength (bottom).

the magnetic field on the sample surfaces, $H_{x}, H_{y}$, and $H_{z}$, can be obtained by solving Eqs. (5)-(7).

\section{EXPERIMENTAL RESULTS}

A cubic sample with the side length of $22.7 \mathrm{~mm}$ and six guard pieces with dimensions of $22 \times 22 \times 5 \mathrm{~mm}^{3}$ in this study were cut from a cylindrical SOMALOYTM 500, a new soft magnetic composite recently developed by Höganäs $\mathrm{AB}$, Sweden. This material is made of highly pure iron powder with surface coating to ensure low eddy current loss. It is developed specifically for applications in electromagnetic devices with 3D fluxes, such as electrical motors, transformers, ignition systems, and sensors. Carefully, the relative positions of the sample and guard pieces were marked during the cutting to ensure the anisotropic axes of the guard pieces align with those of the sample.

Three orthogonal $B$ coils of 30 turns with the doublelayer structure are wound around the cubic sample for measuring the $\mathbf{B}$ components in the $X, Y$, and $Z$ directions. Each $H$ search coil is wound around a $0.5 \mathrm{~mm}$ thick epoxy glass with 110 turns forward and 110 turns backward. The $H$ coils on the opposite sample surfaces are connected in series. To improve the accuracy, $0.05 \mathrm{~mm}$ enameled copper wire is used for the search coils and the connection wires. The connection wires are twisted in order to avoid picking up any unwanted stray fields. The six $H$ search coils are integrated into an $H$ sensing box and sealed by epoxy resin in order to protect the very dedicate wires.

The magnetic flux density components in the sample can be calculated from the emfs across the $B$ coil, $P_{x}, P_{y}$, and $P_{z}$, by

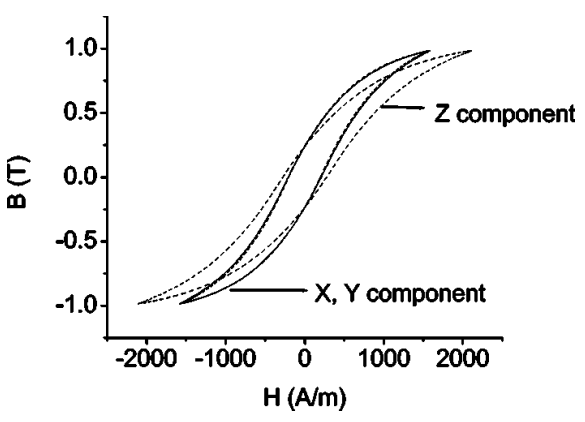

FIG. 4. The hysteresis loops for $X, Y$, and $Z$ components. (Note: $X$ and $Y$ components overlap).

$$
B_{i}=-\frac{1}{A_{i}} \int P_{i} d t,
$$

where $A_{i}(i=x, y$, or $z)$ are the $B$ coil constants for the $X, Y$, and $Z$ directions, which are determined by calibration using same structure coil around a cubic perspex with same cross section with the sample. The constant, in fact, is the product of number of the turns and the area of cross section.

Figure 3 shows the $\mathbf{B}$ and $\mathbf{H}$ vector loci when the three components of $\mathbf{B}$ vector were controlled to be sine wave forms with an amplitude of $1 \mathrm{~T}$ at $50 \mathrm{~Hz}$. Their projections are also plotted. Figure 4 shows the hysteresis loops of the $X$, $Y$, and $Z$ components. The sample is isotropic overall. The properties in the $X$ and $Y$ axes are very similar, but the $Z$ axis seems to be a hard axis. This might be caused by the pressure along $Z$ direction during the processing of formation. The feedback wave form control system had difficulty to maintain sinusoidal wave forms when the $B$ value is set to be close to the saturation value of the magnetic material. However, the $X, Y$, and $Z$ components of saturated hysteresis loop are very similar to the data measured on a circular ring sample provided by the manufacturer.

\section{CONCLUSION}

In conclusion, a 3D magnetic property testing system has been successfully built and calibrated for characterization of soft magnetic materials. A cubic sample of a soft magnetic composite, SOMALOYTM 500, was measured. As shown by its $3 \mathrm{D}$ hysteresis loci, the sample is isotropic overall though the property along the pressure direction is slightly different from the others.

${ }^{1}$ J. G. Zhu, J. J. Zhong, V. S. Ramsden, and Y. G. Guo, J. Appl. Phys. 85, 4403 (1999).

${ }^{2}$ G. Bertotti, A. Canova, M. Chiampi, D. Chiarabaglio, F. Fiorillo, and A. M. Rietto, J. Magn. Magn. Mater. 133, 647 (1994).

${ }^{3}$ L. R. Dupre, F. Fiorillo, C. Appino, A. M. Rietto, and J. Melkebeek, J. Appl. Phys. 87, 6511 (2000).

${ }^{4}$ R. D. Findlay, N. Stranges, and D. K. Mackay, IEEE Trans. Energy Convers. 9, 543 (1994).

${ }^{5}$ S. A. Harrison, R. Street, J. R. Budge, and S. K. Jones, IEEE Trans. Magn. 35, 3962 (1999).

${ }^{6}$ T. Kochmann, J. Magn. Magn. Mater. 160, 145 (1996).

${ }^{7}$ R. Keller and E. Schmidbauer, Geophys. J. Int. 138, 334 (1999).

${ }^{8}$ N. Stranges and R. D. Findlay, IEEE Trans. Magn. 36, 3457 (2000).

${ }^{9}$ J. Sievert, H. Ahlers, M. Enokizono, S. Kauke, L. Rahf, and J. Xu, J. Magn. Magn. Mater. 91, 112 (1992).

${ }^{10}$ V. Basso and G. Bertotti, J. Magn. Magn. Mater. 215, 1 (2000). 